\title{
Combined inhibition of the EGFR and mTOR pathways in EGFR wild-type non-small cell lung cancer cell lines with different genetic backgrounds
}

\author{
YU HUANG, YU CHEN, QIN MEI, YUAN CHEN, SHIYING YU and SHU XIA \\ Cancer Center of Tongji Hospital, Tongji Medical College, \\ Huazhong University of Science and Technology, Wuhan, Hubei 430030, P.R. China
}

Received November 24, 2012; Accepted February 12, 2013

DOI: $10.3892 /$ or.2013.2357

\begin{abstract}
The epidermal growth factor receptor (EGFR) signaling pathway is widely activated in non-small cell lung cancer (NSCLC). However, only a subset of patients with NSCLC is sensitive to EGFR tyrosine kinase inhibitors (TKIs), particularly those with activating EGFR mutations. The mammalian target of rapamycin (mTOR) is another key intracellular kinase that plays an important role in the onset and progression of many types of human cancers and has been proven to be linked with primary resistance to EGFR inhibitors. We performed this study to investigate the combined inhibitory effect of the mTOR inhibitor RAD001 and the EGFR-TKI gefitinib in three EGFR wild-type NSCLC cell lines: A549 (PIK3CA wild-type), NCI-H460 (PIK3CA mutant) and NCI-H661 (PIK3CA wild-type). All cell lines demonstrate a poor response to gefitinib, but have a different genetic status for PIK3CA. We used MTT assays to measure cell proliferation. Flow cytometry was used to assess the effects on apoptosis and cell cycle arrest. Immunoblot analysis was used to evaluate the expression of downstream proteins. Treatment of RAD001 alone showed dose-dependent growth inhibition in all three cell lines. The combination of gefitinib and RAD001 resulted in synergistic growth inhibition in NCI-H460 cells, but only an additive inhibitory effect on A549 and NCI-H661 cells. Exposure to the combination of RAD001 and gefitinib led to a significant reduction in phosphorylated AKT levels in NCI-H460 cells; however, this was not noted in the other two cell lines. In conclusion, our data indicate that the dual inhibition of the EGFR/mTOR pathways may be a promising approach to treat EGFR wild-type NSCLC; however, this may be dependent on the PIK3CA mutation status.
\end{abstract}

Correspondence to: Dr Shu Xia or Dr Shiying Yu, Cancer Center of Tongji Hospital, Tongji Medical College, Huazhong University of Science and Technology, Wuhan, Hubei 430030, P.R. China

E-mail: xiashu1900@yahoo.com.cn

E-mail: syyu@tjh.tjmu.edu.cn

Key words: non-small cell lung cancer, EGFR, mTOR, PIK3CA

\section{Introduction}

Despite recent advances in the understanding of lung cancer biology, non-small cell lung cancer (NSCLC) is still the leading cause of death from cancer in the world (1). Aberrant signaling pathways have been extensively studied in human NSCLC, and targeted therapy has been applied in the clinic. The epidermal growth factor receptor (EGFR) is one of the most successive targets $(2,3)$. The EGFR tyrosine kinase inhibitors (EGFR-TKIs), gefitinib and erlotinib, that specifically target the EGFR tyrosine kinase domain have already been approved by the US FDA as monotherapy for the treatment of patients with locally advanced or metastatic NSCLC, and in many other countries gefitinib has also gained approval for the treatment of advanced NSCLC patients with EGFR mutations in all lines of therapies (2). The majority of NSCLC cases overexpress EGFR, but only a limited subset of patients who harbor EGFR mutations benefit from therapy (1). Intrinsic resistance in patients without EGFR mutations, as well as acquired resistance after the initial response to TKIs, is a serious clinical issue. Therefore, novel therapeutic strategies are under investigation.

To date, various studies have been performed to identify the population of patients that may be responsive to EGFRTKIs. The possible mechanisms that underlie the primary resistance of these drugs have been supposed as follows: i) activation of other signaling pathways alternative to EGFR, such as the insulin-like growth factor 1 receptor (IGF-1R) and vascular endothelial growth factor receptor (VEGFR); and ii) deregulation of downstream signaling pathways such as AKT/mTOR (2-5). Recently, much research has focused on combined treatment with different signaling drugs, representing a novel strategy for cancer therapy (6-8). Here, mTOR inhibitor emerged as an attractive drug candidate due to the role of the mTOR pathway in the development of lung cancer resistance.

The mammalian target of rapamycin (mTOR) is a highly conversed serine-threonine kinase, located in the PI3K-AKT pathway. It controls cell growth, proliferation, protein synthesis, autophagy and metabolism, resulting in its increased attention for use in cancer-targeted therapies (9-11). Various mTOR inhibitors have been used in trials for anti- 
cancer. RAD001 (everolimus) is an orally bioavailable mTOR inhibitor derived from rapamycin, that has gained US FDA approval for the treatment of metastatic renal carcinoma (11). However, in many other clinical trials, it has demonstrated limited success, which may be a result of the feedback activation of the PI3K-AKT targeted pathway $(9,10)$.

Taken together, we proposed that combined treatment of an EGFR-TKI, gefitinib, and an mTOR inhibitor, RAD001, may have more efficacy in treating NSCLC cell lines. Thus, in the present study, we examine the antitumor effect of an mTOR inhibitor combined with gefitinib on EGFR-TKI primary resistant EGFR-wild-type NSCLC cell lines. To elucidate the effects and the underlying mechanisms, we used gefitinib and RAD001 to treat three NSCLC cell lines A549, NCI-H661, NCI-H460 that have wild-type EGFR but have a different mutation status of PIK3CA. Oncogenic mutation of PIK3CA was considered here to evaluate whether there is a genetically distinct response to the combined treatment.

\section{Materials and methods}

Cell culture and reagents. The human NSCLC cell lines, NCI-H460, A549, NCI-H661, were purchased from the Type Culture Collection of the Chinese Academy of Sciences, Shanghai, China. NCI-H460 and NCI-H661 were cultured in RPMI-1640 medium (Gibco) supplemented with 10\% fetal bovine serum. A549 was cultured in F12K medium as recommended. RAD001 (everolimus) was provided by Novartis Pharmaceuticals, and gefitinib was supplied by AstraZeneca. Drugs were dissolved in DMSO (Sigma-Aldrich, St. Louis, MO, USA), respectively, and stored at $-20^{\circ} \mathrm{C}$. Solutions were finally diluted in the culture medium to achieve the required concentrations.

Cell proliferation assay. The effects of the drugs on cell proliferation were determined by a colorimetric assay using 3-(4,5-dimethylthiazol-2-yl)-2,5-diphenyltetrazolium bromide (MTT; Sigma-Aldrich). Cells were plated in 96-well plates at a density of 2,000-4,000 cells and allowed to attach overnight. The next day, cells were treated with a range of drug concentrations of gefitinib, RAD001 or the combination as recommended. Control cells were treated with the same concentration of the vehicle DMSO.

After a 72-h incubation, $10 \mu \mathrm{l}$ MTT solution was added to each well. Four hours later, formazan crystals were solubilized in $100 \mu 1 \mathrm{DMSO}$, and the absorbance was measured at $490 \mathrm{~nm}$.

Combined effect analysis. The combined effect of gefitinib and RAD001 was determined by using the Bliss additivism model. The Bliss value is expressed as an equation Ebliss = EA + $\mathrm{EB}-\mathrm{EA} \times \mathrm{EB}$, where EA and EB are the fractional inhibition effects of drug $\mathrm{A}$ and $\mathrm{B}$ alone at an indicated concentration, respectively. Ebliss is the fractional inhibition that would be expected if the combination of the two drugs was exactly additive. If the experimentally measured fractional inhibition of combined treatment is more than the expected Ebliss value, then the combination is considered to be synergistic, while if the experimentally combined effect is less than the expected
Table I. EGFR and PIK3CA mutation status of the selected cell lines.

\begin{tabular}{llll}
\hline Cell lines & PIK3CA & PTEN & EGFR \\
\hline A549 & Wild-type & Wild-type & Wild-type \\
NCI-H661 & Wild-type & Wild-type & Wild-type \\
NCI-H460 & Mutant & Wild-type & Wild-type \\
\hline
\end{tabular}

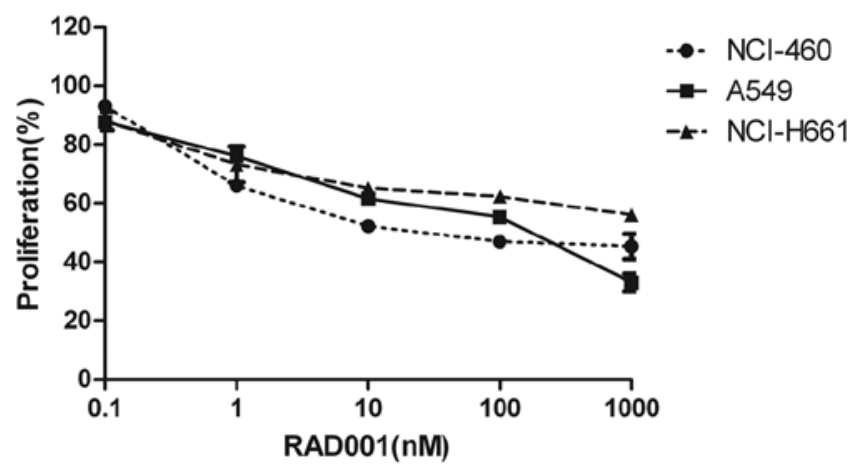

Figure 1. RAD001 is effective in inhibiting the growth of gefitinib-resistant cells. NCI-H460, A549 and NCI-H661 cells were treated with RAD001 alone at concentrations from 0.1 to $1000 \mathrm{nM}$ for $72 \mathrm{~h}$. Dose-dependent growth inhibitory effects of RAD001 are shown. Data were obtained from three experiments, and the bars represent the standard deviation (SD).

Ebliss value, then the interaction is antagonistic. Otherwise, the combined effect is additive $(12,13)$.

Apoptosis and cell cycle analysis. All cells were seeded at $1 \times 10^{5} /$ well in 6 -well plates and allowed to attach. Then RAD001, gefitinib or their combination was added at the indicated concentrations. After a 48-h incubation, the cells were harvested. For apoptosis analysis, harvested cells were stained with Annexin V-fluorescein isothiocyanate (FITC) and propidium iodide (PI) using a commercial kit (FITC apoptosis kit, Nanjing KeyGen Biotech., Co., Ltd., Nanjing, China). The fluorescence levels of apoptotic cells were determined by flow cytometry on a FACSort, (BD Biosciences, Franklin Lakes, NJ, USA) and analyzed using the CellQuest software. To analyze the cell cycle distribution, cell nuclear DNA was stained with PI and detected with FACSort flow cytometry. Cell cycle profile was performed using ModFit LT software (BD Biosciences).

Western blot analysis. Cells were cultured for $24 \mathrm{~h}$ and stimulated with inhibitors at the indicated concentrations. Protein extracts were normalized according to the protein content, resolved by $10 \%$ SDS-PAGE, transferred to nitrocellulose membranes, probed overnight at $4{ }^{\circ} \mathrm{C}$ with antibodies and finally revealed using the ECL western blot analysis system. The following antibodies were used: anti-phospho-AKT (S473) and anti-phospho-p70S6K (Thr389) were obtained from Cell Signaling Technology. Anti-AKT and anti-p70S6K was purchased from Epitomics. Anti- $\beta$-actin and horseradish peroxidase-conjugated anti-rabbit or anti-mouse IgG were 

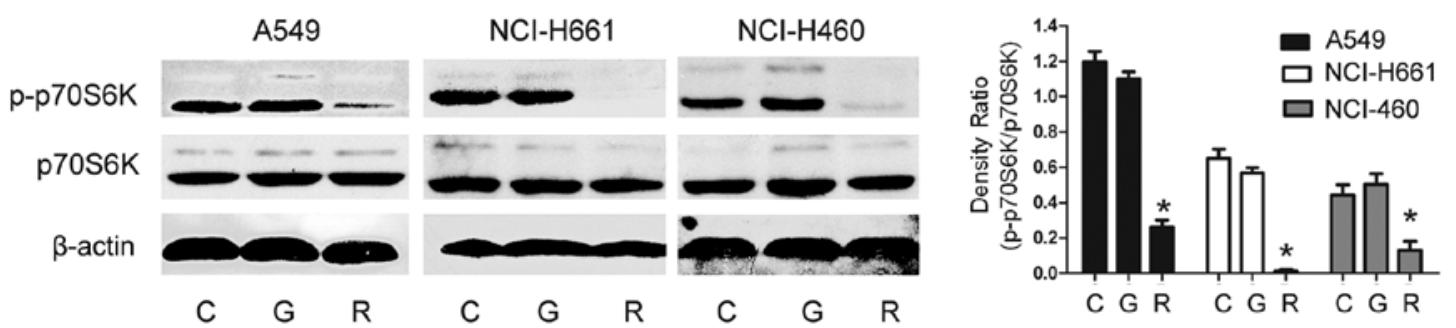

Figure 2. Effects of gefitinib and RAD001 on the phosphorylation of p70S6K. All of the cell lines were incubated as recommended and were treated with gefitinib $(1 \mu \mathrm{M})$ or RAD001 $(10 \mathrm{nM})$ for $24 \mathrm{~h}$. Whole cell lysates were subjected to western blot analysis. Phospho-p70S6K was probed. While exposure to gefitinib did not decrease the phosphorylation level of the downstream effector ofthe mTOR pathway, p70S6K, RAD001 treatment significantly inhibited p70S6K phosphorylation in all of the cell lines studied. The blots displayed were repeated three times. "P<0.01 vs. control. C, DMSO control; G, gefitinib; R, RAD001.
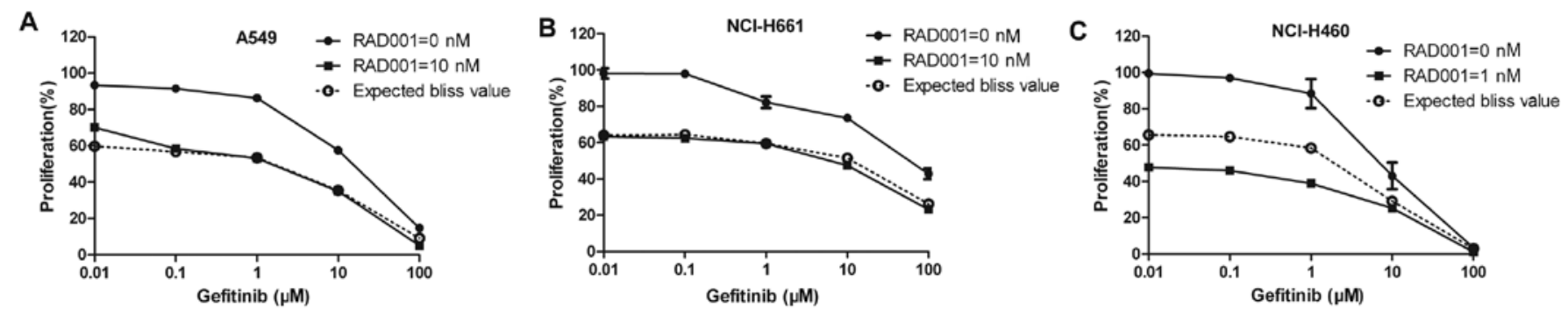

Figure 3. Combination of RAD001 and gefitinib exerts a synergistic effect on the cell growth of NCI-H460 cells. (A) A549, (B) NCI-H661 and (C) NCI-H460 cells were treated with gefitinib alone at a range of concentrations from 0.01 to $100 \mu \mathrm{M}$ or concomitant with RAD001 at a fixed concentration (1 to $10 \mathrm{nM}$, depending on the cell line) for $72 \mathrm{~h}$. Proliferation curves of the cells treated with gefitinib alone or the combined treatment vs. the theoretical Bliss curve (dashed line) are shown. Dashed line, Bliss additivity curve representing the theoretical expectation if the combined effects were exactly additive. A synergistic inhibitory effect was noted only in NCI-H460 cells. Data were obtained from three experiment and are expressed as mean values \pm SD.

purchased from Boster Biological Technology (Wuhan, China). Each sample was assayed in triplicate.

Statistics. The statistical significance between two different groups was evaluated by the Student's t-test. SPSS 13.0 was performed for all the statistical tests. All P-values were two-sided; $\mathrm{P}<0.05$ was considered to indicate a statistically significant result. All data are presented as means \pm SD.

\section{Results}

Effects of single-drug treatment with gefitinib or RADO01 on cell proliferation. We determined the effects of gefitinib treatment alone on three NSCLC cell lines with wild-type EGFR (NCI-H460, A549 and NCI-H661). First, we compared the proliferation rate of these cell lines following treatment with gefitinib. Cells were treated with gefitinib at a range of concentrations for $72 \mathrm{~h}$, and the median inhibitory concentration $\left(\mathrm{IC}_{50}\right)$ values were determined by MTT proliferation assay. $\mathrm{IC}_{50}$ values were 8,18 and $>20 \mu \mathrm{M}$ for NCI-H460, A549 and NCI-H661, respectively. According to previous studies $(1,14)$, cells having an $\mathrm{IC}_{50}>1 \mu \mathrm{M}$ are considered as being resistant to gefitinib. Therefore, the three NSCLC cell lines had primary resistance to gefitinib. The cell lines exhibited wild-type EGFR but had a different mutation status of PIK3CA (Table I), suggesting that the EGFR mutation status plays an important role in the determination of gefitinib resistance.

To determine the effects of the mTOR inhibitor RAD001 on these gefitinib-resistant cells, we also performed a proliferation assay. We demonstrated that RAD001 treatment caused a mild but significant growth inhibition in all cell lines studied (Fig. 1). Specially, following a 72-h treatment, $100 \mathrm{nM}$ of RAD001 achieved a mild reduction in the percentage of viable A549 ( 51\%), NCI-H460 ( 55\%) and NCI-H661 cells $(\sim 38 \%)$, and the inhibitory effects were dose-dependent. However, a cell killing effect of $>75 \%$ was not observed even at concentrations up to $1,000 \mathrm{nM}$, which support the need for alternative regimens to improve monotherapeutic efficacy. Notably, expression of wild-type EGFR did not affect the responsiveness of these cells to RAD001. To further explore the effect of RAD001 on mTOR signaling, we performed a western blot assay. P70S6K is an important downstream effector of mTOR, and its phosphorylation has been reported to be effectively inhibited by rapamycin analog treatment (15). In the present study, western blot assay was performed after exposure to RAD001 at a concentration of $10 \mathrm{nM}$ for $24 \mathrm{~h}$, showing a significant decrease in the phosphorylation of p70S6K at Thr389 in all cell lines (Fig. 2). Therefore, these results indicate the importance of the mTOR signaling pathway in the proliferation of NSCLC cells, suggesting the need for combined treatment to enhance the antitumor effect.

Gefitinib cooperates with RAD001 to enhance the inhibitory effect of either drug alone in NCI-H460 cells with PIK3CA mutation. To examine the inhibitory effect following suppression of both EGFR and mTOR pathways, we evaluated the combined effect of gefitinib and RAD001 on growth inhibition, apoptosis and cell cycle arrest. MTT assay was performed on cells treated with gefitinib at concentrations ranging from 0.01 to $100 \mu \mathrm{M}$, in the presence of RAD001 at fixed concentrations 
A

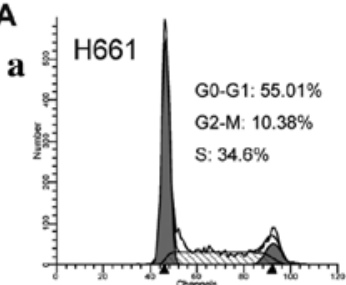

Control

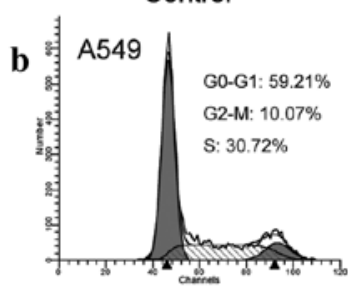

Control

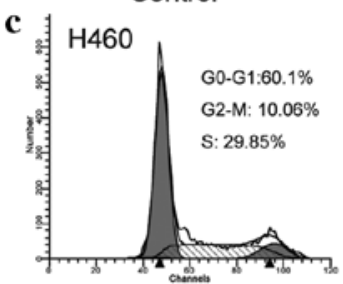

Control

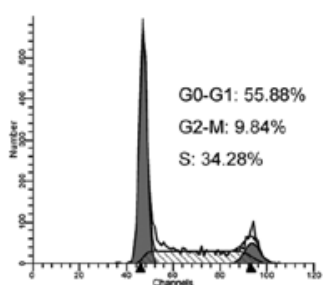

Gefitinib



Gefitinib

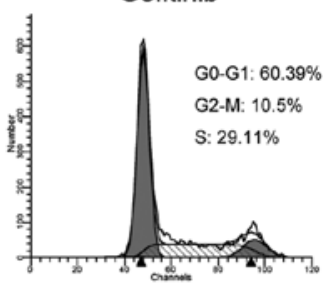

Gefitinib

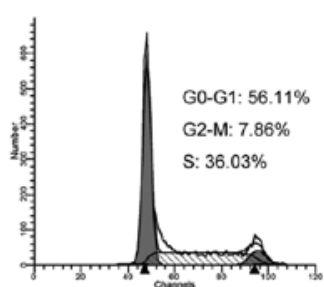

RAD001

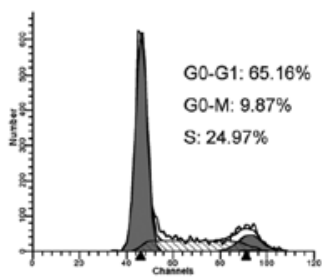

RAD001

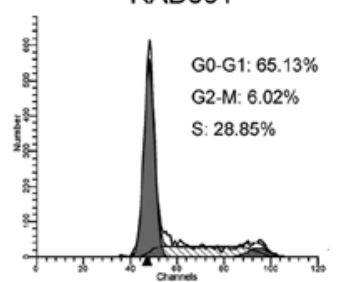

RAD001

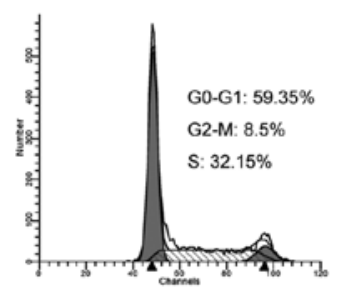

Combined

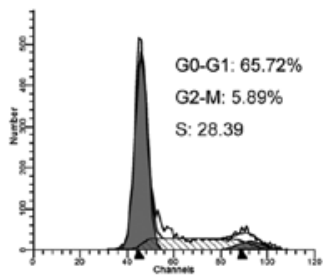

Combined

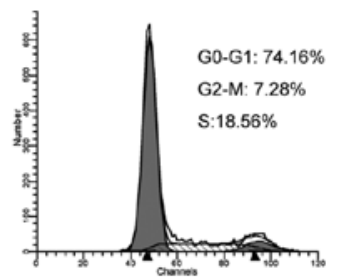

Combined
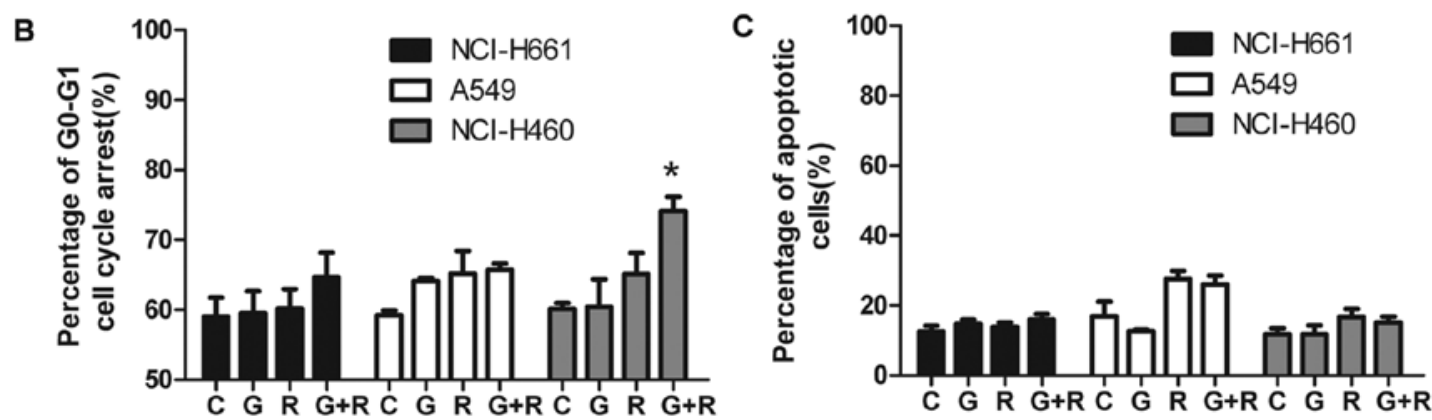

Figure 4. Combination of RAD001 and gefitinib induces enhanced cell cycle arrest (G0-G1 phase). The indicated cell lines were treated with gefitinib (1 $\mu \mathrm{M})$, RAD001 (10 nM) alone or their combination for $48 \mathrm{~h}$. The cells were then harvested for (A and B) cell cycle analysis and for (C) assessment of apoptosis by staining with Annexin V-FITC using flow cytometry. (A) The effects of different treatments on the cell cycle distribution of (a) NCI-H661, (b) A549 and (c) NCI-H460 cells. Values in B represent the percentage of cells undergoing G0-G1 phase arrest. The difference in the percentage of cells in G0-G1 phase arrest between the monotherapy and combined treatments was statistically significant in the NCI-H460 cells ("P $<0.05$ for gefitinib combined with RAD001 vs. control, gefitinib or RAD001 alone). Results for the other two cell lines were not statistically significant. Values in C represent the percentage of apoptotic cells as the sum of cells in early and late stage of apoptosis. No significantly enhanced apoptosis was observed in all studied cell lines. C, DMSO control; G, gefitinib; R, RAD001; G+R, combined treatment with gefitinib and RAD001.

from 1 to $10 \mathrm{nM}$, depending on the cell line. In an attempt to evaluate the combined efficacy, we used the Bliss additivism model to clarify whether the effect was additive, synergistic or antagonistic as described in Materials and methods. The proliferation curve of the cells following treatment with varying concentrations of gefitinib in the presence or absence of a fixed concentration of RAD001 are shown in Fig. 3. To note, the Bliss theoretical curves that would be expected if the combination was additive was calculated using the Bliss equation and are shown in Fig. 3. Comparing the experimental and the theoretical curves, only in the NCI-H460 cells was the experimentally measured fractional inhibition of the combined treatment more than the expected Ebliss value. Therefore, the effect of the combination treatment on the NCI-H460 cells was synergistic. The effects on the other two cell lines, A549 and NCI-H661, showed pure additivity. Of note, NCI-H460 cells harbor a PI3K mutation, whereas A549 and NCI-H661 harbor no mutation in this gene.

For further investigation, we explored the effects of the combined treatment on the percentage of cell apoptosis and cell cycle distribution following treatment with two single drugs and their combination using flow cytometric analyses of Annexin $\mathrm{V}$ binding. The combination treatment did not enhance the rate of apoptosis when compared with this rate following treatment with either drug alone in all of the cell lines studied (Fig. 4C). In addition, we tested the cell cycle profiles of the three cell lines after treatment with each of the drugs alone or their combination (Fig. 4A and B). We 

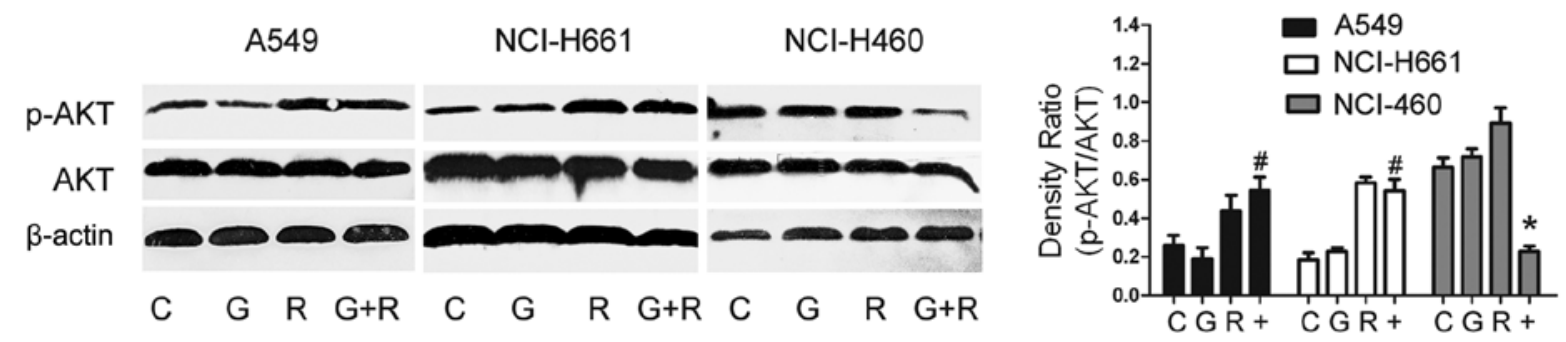

Figure 5. Effects of the combination of gefitinib and RAD001 on AKT/mTOR signaling. Cells were treated with RAD001 (10 nM) or gefitinib (1 $\mu \mathrm{M})$ alone or in combination. After $24 \mathrm{~h}$, cells were harvested and lysed for western blot analysis with the indicated antibodies. Immunoblotting showed suppression of the p-AKT level in NCI-H460 cells after the combination treatment with gefitininb and RAD001, but not in the other two cell lines. *P<0.01 for gefitinib combined with RAD001 vs. control, gefitinib or RAD001 alone. "P>0.05 for gefitinib combined with RAD001 vs. RAD001 alone. C, DMSO control; G, gefitinib; R, RAD001; G+R, combined treatment with gefitinib and RAD001.

found that the treatment with the combination of gefitinib and RAD001 had a great impact on the cell cycle distribution in the NCI-H460 cells. The combination caused accumulated of cell cycle arrest in the G0/G1 phase, and the proportion of cells in the $\mathrm{S}$ and $\mathrm{G} 2 / \mathrm{M}$ phase decreased following treatment with $1 \mu \mathrm{M}$ gefitinib and $10 \mathrm{nM}$ RAD001 in combination for $48 \mathrm{~h}$; the proportion of cells in the G0/G1 phase increased from 60.10 to $74.16 \%(\mathrm{P}<0.05)$ (Fig. 4A), indicating that RAD001 enhanced a cytostatic effect, rather than a cytotoxic effect. However, no significant changes were detected in the NCI-H661 and A549 cells (Fig. 4A and B).

Combination treatment diminishes AKT signaling in the NCI-H460 cells. To further explore the mechanisms of genotype-dependent proliferation after the indicated treatments, we evaluated therapy-associated changes in cell signaling pathway. Since AKT has been proven to play an important role in cell survival and is highly linked with mTOR, we aimed to ascertain how AKT phosphorylation and the mTOR downstream effector are influenced following treatment with gefitinib and RAD001 alone or in combination (Fig. 5). As we previously reported (Fig. 2), western blot analysis demonstrated that exposure to RAD001 at a concentration of $10 \mathrm{nM}$ induced a significant decrease in the phosphorylation level of p70S6K, an important downstream effector of mTOR, in all the cell lines studied. Yet, no changes were observed following treatment with gifitinib alone, which partially supports the idea of combined inhibition with mTOR. Although AKT phosphorylation is highly suppressed by gefitinib in sensitive cell lines (8), it persistently remained the same after treatment with gefitinib alone at a concentration of $1 \mu \mathrm{M}$ in the gefitinib primary resistant NSCLC cell lines (Fig. 5). RAD001 and other rapamycin analogs have been shown to indirectly activate mTORC2-dependent AKT phosphorylation at Ser473 (15). We found that the p-AKT level was increased after treatment with RAD001 alone at a concentration of $10 \mathrm{nM}$, consistent with various studies due to a negative feedback loop after inhibition of s6k phosphorylation $(10,15)$.

Therefore, we investigated whether the phosphorylation level of AKT is influenced by the combination treatment. Exposure to the combination of RAD001 (10 nM) and gefitinib $(1 \mu \mathrm{M})$ led to a significant reduction in $\mathrm{p}$-AKT level in the NCI-H460 cells (PIK3CA mutant), when compared with the respective gefitinib or RAD001 treatment. However, no reduc- tion in AKT phosphorylation was noted after combination treatment in the 549 and NCI-H661 cells, which is consistent with their additive combined effects.

\section{Discussion}

Non-small cell lung cancer represents $80 \%$ of all lung cancer cases, and the majority of patients present with locally advanced or metastatic disease. Treating advanced NSCLC patients with platinum-based combination chemotherapy has achieved more success than best supportive care. However, it yields a median survival $<12-13$ months (6). Novel therapeutic strategies including molecular targeted therapy such as EGFRTKIs have been proven to be effective, but only limited to a subset of patients $(1,2)$. Signaling-based combination therapies have recently emerged as novel attractive strategies $(6,8,16)$. Evidence supports the deregulation of the PI3K/mTOR pathway as a possible mechanism for primary resistance of NSCLC to EGFR-TKIs $(2,17)$. Therefore, this study was performed using three EGFR-TKI-resistant cell lines to evaluate the therapeutic potential of the concomitant inhibition of EGFR and mTOR signaling. The studied cell lines are characterized by expression of wild-type EGFR, but with a different genetic status of PIK3CA.

Our present study demonstrated that these three cell lines all exhibit a poor response to gefitinib, and the mTOR pathway is required for sustained growth in EGFR-TKI-resistant cells. All of the cells harbored wild-type EGFR, which is consistent with the role of the EGFR mutational status in predicting EGFR-TKI efficacy. Phosphorylation of p70S6K, an important downstream factor of mTOR, was not inhibited by gefitinib alone in the three resistant cells, which suggests that maintained activation of this signaling pathway was associated with gefitinib intrinsic resistance. Indeed, the role of mTOR in tumorigenesis has already been intensely investigated, and research has previously revealed that constructive activation of the AKT/mTOR pathway is present in a defined subset of NSCLC cases (18). Furthermore, we found that the mTOR inhibitor RAD001 induced growth inhibition in gefitinib primary-resistant cells, and p70S6K was effectively reduced. However, it is known that RAD001 is a cytostatic factor and cannot fully inhibit cell proliferation when a certain percentage of inhibition is achieved. Inhibition with mTOR inhibitor rapamycin or its derivates can indirectly activate 
AKT phosphorylation through a feedback pathway by IRS or Grb (19-21), which may partially compensate for the blockage of p70S6K. Here, p-AKT was shown to be increased in RAD001-treated cells, which may be a possible explanation for its cytostatic effect and limited therapeutic efficacy following treatment with RAD001 alone.

However, rapamycin or its analogs were found to be able to synergize drug sensitivity in many cases using combined treatment $(22,23)$. Thus, combined blockage of both EGFR and mTOR is proposed to exhibit a more effective antitumor effect. This hypothesis has been validated in many human cancer cell lines. It has already been reported that inhibition of the mTOR pathway by RAD001 produced a cooperative effect with EGFR inhibitors and overcame resistance to anti-EGFR drugs according to Bianco et al (8). Moreover, combined inhibition using an mTOR inhibitor and anti-EGFR drugs was also found to be synergistic in selected NSCLC cells or patients in many other studies. Higher proliferation index or KRAS mutated status were included $(24,25)$. In our study, the genetic status of PIK3CA was considered to illustrate the role of genetically distinct combined therapeutic interventions in EFGR-wild-type cells. Thus, we performed this study in three NSCLC cell lines A549 (PIK3CA wild-type), NCI-H661 (PIK3CA wild-type) and NCI-H460 (PIK3CA mutant), which all express wild-type EGFR. We aimed to ascertain whether RAD001 enhances the antitumor effect of gefitinib in these cells, and whether cells with a genetically distinct status of PIK3CA showed a different efficacy. Here, we demonstrated that concomitant therapy with gefitinib and RAD001 had a significant synergistic inhibitory effect on the NSCLC cell line NCI-H460 with PIK3CA mutation, but was not effective on cells with wild-type PIK3CA, according to the Bliss additivism model. Moreover, consistent with this, cell cycle distribution was significantly altered in the NCI-H460 cells. We showed that the drug combination promoted extensive accumulation of cells in the G0-G1 phase of the cell cycle, while the percentages of cells in the $\mathrm{S}$ and G0/M phase was decreased. The same phenomenon was not noted in the A549 and NCI-H661 cells. Notably, we did not observe significant apoptosis following any of the treatments, which may be consistent with the cytostatic effect of RAD001.

The mechanism responsible for the different efficacy in NCI-H460 cells compared to the other two cell lines may be associated with their PIK3CA mutation status. Oncogenic mutations of the PI3K catalytic a subunit (PIK3CA) are among the most frequently reported genetic aberrations in many human epithelial cancers (15), and it seems to be an important indicator of resistance and poor outcome in patients with NSCLC treated with EGFR-TKIs (26). Furthermore, oncogenic mutation of PIK3CA may lead to constitutively activity of downstream signaling such as the PI3K/AKT/mTOR pathway, which is partially EGFR independent (27). One recent study reported that the combination of EGFR monoclonal antibodies with a PI3K inhibitor was a good therapeutic option in PIK3CA mutated head and neck squamous cell cancer (28). Therefore, combined treatment with EFGR-TKI and RAD001 is proposed to effectively inhibit cell proliferation in cells with PIK3CA mutation. Our research did not show synergistic inhibition of the combination treatment in the PIK3CA wild-type A549 and NCI-H661 cell lines, suggesting that another receptor, tyrosine kinase pathway may be activated in these cells resulting in AKT activation and cannot be reduced by combined treatment. Tamburini et al (29) strongly indicated an mTOR-independent deregulation of oncogenic protein synthesis in human myeloid leukemogenesis. In addition, consistent with this, it has been shown that an EGFR-TKI and RAD001 can effectively inhibit AKT phosphorylation in resistant cells with a PI3KCA mutation. In the present study, combination treatment disturbed the $\mathrm{PI} 3 \mathrm{~K} / \mathrm{mTOR}$ pathway and enhanced the antitumor effect of monotherapy on NCI-H460 cells.

It is critically important to develop specific biomarkers for predicting a certain response of targeted cancer therapies (30). Here, our results demonstrated that the combination therapy was effective only in selected cells, which may harbor mutations of the PIK3CA gene.

Recently, combined inhibition of EGFR and mTOR has been previously investigated in patients with advanced NSCLC, and demonstrated a clinical response in KRAS- mutated cancer patients, although the study was not concerned with PI3KCA mutations (24). PIK3CA mutations can be widely noted in many types of human cancers. Due to its contribution in cancer pathogenesis, therapy targeting this pathway has been proven to be effective (15). In the present study, we further confirmed that combined inhibition with an EGFR-TKI and an mTOR inhibitor effectively induced cell proliferation inhibition and cell cycle arrest in EGFR-wild-type NSCLC cells with a PIK3CA mutation, disturbing AKT signaling. Thus, further study of the different PI3KCA mutation status in predicting the efficacy of the concomitant inhibition of the EGFR and mTOR pathways in NSCLC is warranted. Combined inhibition of the EGFR and mTOR pathways may provide an excellent therapeutic option for treatment of PIK3CA-mutated EFGRwild-type NSCLC.

\section{Acknowledgements}

This study was supported by a grant from the Wujieping Foundation, China.

\section{References}

1. Sharma SV, Bell DW, Settleman J and Haber DA: Epidermal growth factor receptor mutations in lung cancer. Nat Rev Cancer 7: 169-181, 2007.

2. Wheeler DL, Dunn EF and Harari PM: Understanding resistance to EGFR inhibitors - impact on future treatment strategies. Nat Rev Clin Oncol 7: 493-507, 2010.

3. Han SW, Kim TY, Hwang PG, et al: Predictive and prognostic impact of epidermal growth factor receptor mutation in non-small cell lung cancer patients treated with gefitinib. J Clin Oncol 23: 2493-2501, 2005.

4. Chakravarti A, Loeffler JS and Dyson NJ: Insulin-like growth factor receptor I mediates resistance to anti-epidermal growth factor receptor therapy in primary human glioblastoma cells through continued activation of phosphoinositide 3-kinase signaling. Cancer Res 62: 200-207, 2002.

5. Engelman JA, Janne PA and Mermel C: ErbB-3 mediates phosphoinositide 3-kinase activity in gefitinib-sensitive non-small cell lung cancer cell lines. Proc Natl Acad Sci USA 102: 3788-3793, 2005.

6. Gorzalczany Y, Gilad Y, Amihai D, Hammel I, Sagi-Eisenberg R and Merimsky O: Combining an EGFR directed tyrosine kinase inhibitor with autophagy-inducing drugs: a beneficial strategy to combat non-small cell lung cancer. Cancer Lett 310: 207-215, 2011. 
7. Morrow PK, Wulf GM, Ensor J, et al: Phase I/II study of trastuzumab in combination with everolimus (RAD001) in patients with HER2-overexpressing metastatic breast cancer who progressed on trastuzumab-based therapy. J Clin Oncol 29: 3126-3132, 2011.

8. Bianco R, Garofalo S, Rosa R, Damiano V, Gelardi T, Daniele G, Marciano R, Ciardiello F and Tortora G: Inhibition of mTOR pathway by everolimus cooperates with EGFR inhibitors in human tumours sensitive and resistant to anti-EGFR drugs. $\mathrm{Br} \mathrm{J}$ Cancer 98: 923-930, 2008.

9. Zoncu R, Efeyan A and Sabatini DM: mTOR: from growth signal integration to cancer, diabetes and ageing. Nat Rev Mol Cell Biol 12: 21-35, 2010.

10. Yea SS and Fruman DA: Cell signaling. New mTOR targets Grb attention. Science 332: 1270-1271, 2011.

11. Atkins MB, Yasothan U and Kirkpatrick P: Everolimus. Nat Rev Drug Discov 8: 535-536, 2009.

12. Mordant P, Loriot Y, Leteur C, et al: Dependence on phosphoinositide 3-kinase and RAS-RAF pathways drive the activity of RAF265, a novel RAF/VEGFR2 inhibitor, and RAD001 (Everolimus) in combination. Mol Cancer Ther 9: 358-368, 2010

13. Goldoni M and Johansson C: A mathematical approach to study combined effects of toxicants in vitro: evaluation of the Bliss independence criterion and the Loewe additivity model. Toxicol In Vitro 21: 759-769, 2007.

14. Noro R, Gemma A, Kosaihira S, et al: Gefitinib (Iressa) sensitive lung cancer cell lines show phosphorylation of Akt without ligand stimulation. BMC Cancer 6: 277, 2006.

15. Di Nicolantonio F, Arena S, Tabernero J, et al: Deregulation of the PI3K and KRAS signaling pathways in human cancer cells determines their response to everolimus. J Clin Invest 120 : 2858-2866, 2010.

16. Balko JM, Jones BR, Coakley VL and Black EP: Combined MEK and EGFR inhibition demonstrates synergistic activity in EGFR-dependent NSCLC. Cancer Biol Ther 8: 522-530, 2009.

17. Janmaat ML, Kruyt FA, Rodriguez JA and Giaccone G: Response to epidermal growth factor receptor inhibitors in non-small cell lung cancer cells: limited antiproliferative effects and absence of apoptosis associated with persistent activity of extracellular signal-regulated kinase or Akt kinase pathways. Clin Cancer Res 9: 2316-2326, 2003

18. Dobashi Y, Suzuki S, Kimura M, et al: Paradigm of kinase-driven pathway downstream of epidermal growth factor receptor/Akt in human lung carcinomas. Human Pathol 42: 214-226, 2011.

19. Yu Y, Yoon SO, Poulogiannis G, et al: Phosphoproteomic analysis identifies Grb10 as an mTORC1 substrate that negatively regulates insulin signaling. Science 332: 1322-1326, 2011.
20. Hsu PP, Kang SA, Rameseder J, et al: The mTOR-regulated phosphoproteome reveals a mechanism of mTORC1-mediated inhibition of growth factor signaling. Science 332: 1317-1322, 2011.

21. Shi Y, Yan H, Frost P, Gera J and Lichtenstein A: Mammalian target of rapamycin inhibitors activate the AKT kinase in multiple myeloma cells by up-regulating the insulin-like growth factor receptor/insulin receptor substrate-1/phosphatidylinositol 3-kinase cascade. Mol Cancer Ther 4: 1533-1540, 2005.

22. Goudar RK, Shi Q, Hjelmeland MD, et al: Combination therapy of inhibitors of epidermal growth factor receptor/vascular endothelial growth factor receptor 2 (AEE788) and the mammalian target of rapamycin (RAD001) offers improved glioblastoma tumor growth inhibition. Mol Cancer Ther 4: 101-112, 2005.

23. Gemmill RM, Zhou M, Costa L, Korch C, Bukowski RM and Drabkin HA: Synergistic growth inhibition by Iressa and rapamycin is modulated by VHL mutations in renal cell carcinoma. Br J Cancer 92: 2266-2277, 2005.

24. Price KA, Azzoli CG, Krug LM, et al: Phase II trial of gefitinib and everolimus in advanced non-small cell lung cancer. J Thorac Oncol 5: 1623-1629, 2010.

25. La Monica S, Galetti M, Alfieri RR, et al: Everolimus restores gefitinib sensitivity in resistant non-small cell lung cancer cell lines. Biochem Pharmacol 78: 460-468, 2009.

26. Vienna L, Fortunato B, Lorenza P, et al: Phosphoinositide3-kinase catalytic alpha and KRAS mutations are important predictors of resistance to therapy with epidermal growth factor receptor tyrosine kinase inhibitors in patients with advanced non-small cell lung cancer. J Thorac Oncol 6: 707-715, 2011.

27. Elizabeth B, Alexandra E, Eric B, et al: Rapamycin synergizes with the epidermal growth factor receptor inhibitor erlotinib in non-small cell lung, pancreatic, colon, and breast tumors. Mol Cancer Ther 5: 2627-2684, 2006.

28. Magali R, Paul P, Amelie D, et al: Mechanisms underlying resistance to cetuximab in the HNSCC cell line: role of AKT inhibition in by passing this resistance. Int J Oncol 38: 189-200, 2011.

29. Tamburini J, Green AS and Bardet V: Protein synthesis is resistant to rapamycin and constitutes a promising therapeutic target in acute myeloid leukemia. Blood 114: 1618-1627, 2009.

30. Mohseni M and Park BH: PIK3CA and KRAS mutations predict for response to everolimus therapy: now that's RAD001. J Clin Invest 120: 2655-2658, 2010. 